\title{
Ginseng Berry Extract Rich in Phenolic Compounds Attenuates Oxidative Stress but not Cardiac Remodeling post Myocardial Infarction
}

\author{
Mihir Parikh 1,2,3,+ , Pema Raj 1,3,+ , Liping Yu 1,4 , Jo-Ann Stebbing 1,4, Suvira Prashar 1,4, \\ Jay C. Petkau 1,4, Paramjit S. Tappia 5 (D), Grant N. Pierce 1,2,3, Yaw L. Siow 1,3,4, Dan Brown 1,4, \\ Heather Blewett $1,3,4,6$ (D) and Thomas Netticadan $1,3,4, *$ \\ 1 Canadian Centre for Agri-Food Research in Health and Medicine, St. Boniface Hospital Albrechtsen \\ Research Centre, Winnipeg, MB R2H2A6, Canada; mparikh@sbrc.ca (M.P.); praj@sbrc.ca (P.R.); \\ lyu@sbrc.ca (L.Y.); jstebbing@sbrc.ca (J.-A.S.); sprashar@sbrc.ca (S.P.); jpetkau@sbrc.ca (J.C.P.); \\ gpierce@sbrc.ca (G.N.P.); csiow@sbrc.ca (Y.L.S.); danielbrown500@gmail.com (D.B.); hblewett@sbrc.ca (H.B.) \\ 2 Institute of Cardiovascular Sciences, St. Boniface Hospital Albrechtsen Research Centre, Max Rady College \\ of Medicine, Rady Faculty of Health Sciences, University of Manitoba, Winnipeg, MB R2H2A6, Canada \\ 3 Department of Physiology and Pathophysiology, University of Manitoba, Winnipeg, MB R3E0J9, Canada \\ 4 Agriculture and Agri-Food Canada, Winnipeg, MB R2H2A6, Canada \\ 5 Asper Clinical Research Institute \& Office of Clinical Research, St. Boniface Hospital, Winnipeg, \\ MB R2H2A6, Canada; ptappia@sbrc.ca \\ 6 Department of Food and Human Nutritional Sciences, University of Manitoba, Winnipeg, \\ MB R3T2N2, Canada \\ * Correspondence: tnetticadan@sbrc.ca \\ $\dagger$ These authors contributed equally to this work.
}

Received: 22 December 2018; Accepted: 20 February 2019; Published: 24 February 2019 updates

\begin{abstract}
The cardioprotective effects of ginseng root extracts have been reported. However, nothing is known about the myocardial actions of the phenolic compounds enriched in ginseng berry. Therefore, this study was undertaken to investigate the effects of American ginseng berry extract (GBE) in an experimental model of myocardial infarction (MI). Coronary artery ligation was performed on Sprague-Dawley male rats to induce MI after which animals were randomized into groups receiving either distilled water or GBE intragastrically for 8 weeks. Echocardiography and assays for malondialdehyde (MDA) and TNF- $\alpha$ were conducted. Flow cytometry was used to test the effects of GBE on T cell phenotypes and cytokine production. Although GBE did not improve the cardiac functional parameters, it significantly attenuated oxidative stress in post-MI rat hearts. GBE treatment also resulted in lower than control levels of TNF- $\alpha$ in post-MI rat hearts indicating a strong neutralizing effect of GBE on this cytokine. However, there was no effect of GBE on the proportion of different $\mathrm{T}$ cell subsets or ex-vivo cytokine production. Taken together, the present study demonstrates GBE reduces oxidative stress, however no effect on cardiac structure and function in post-MI rats. Moreover, reduction of TNF- $\alpha$ levels below baseline raises concern regarding its use as prophylactic or preventive adjunct therapy in cardiovascular disease.
\end{abstract}

Keywords: Panax quinquefolius; ginseng berry; myocardial infarction; phenolic compounds

\section{Introduction}

Ginseng has been used for centuries in the traditional medicines in Asia. It is a herb derived from genus Panax, of family Araliaceae and has thirteen different species which are indigenous to Asia and North America [1]. Active components of ginseng include ginsenosides, saponins, polysaccharides, alkaloids, peptides, polyacetylenes, phenolics, and fatty acids [2,3]. 
Out of all the bioactive compounds of ginseng, ginsenosides have been more extensively studied compared to the phenolic compounds. However, phenolic compounds are now being investigated in several studies for their diverse biological actions [3]. Salicylic acid, p-coumaric acid, ferulic acid, cinnamic acid, and quercetin are some of the phenolic compounds identified in ginseng [3]. Unlike ginseng roots, the chemical composition of ginseng berry is less known. A comprehensive profile of the phenolic compounds found in Korean ginseng berry, root, and leaf has been reported [3]. In the Korean ginseng berry, chlorogenic acid was reported to be the predominant compound present, followed by gentisic acid and rutin [3]. In the North American ginseng berry, caffeic acid and chlorogenic acid were reported to be the active polyphenolic constituents in a study of the protective effect of ginseng berry extract against oxidant injury in cardiomyocytes [4]. In spite of reports of the presence of bioactives in ginseng berry, it is not commercially used and is often discarded as a 'useless by-products' $[5,6]$.

A recent study showed that ginseng berry has higher total phenol content (including quercetin, rutin, and resveratrol) than the root [5]. Although both ginseng root and berry have pharmacological actions, in some instances berry has been found to be more effective. Ginseng berry has been reported to have a more potent antihyperglycemic action than the root at the same dose [7]. Ginseng berry has been shown to reduce coagulation of blood [8], improve insulin sensitivity [9], and regulate glucose metabolism [10]. Ginseng berry extract (GBE) was found to protect cardiomyocyte against oxidative stress by activating the antioxidant Nrf2 pathway [11]. An echocardiography study using ginseng root extract demonstrated a significant improvement in left ventricular function [12]. However, ginseng berry with its high phenolic content has not been evaluated for its effect on cardiac structure and function. Accordingly, the present study investigated the effect of a phenolic rich GBE on cardiac structure and function. Furthermore, the damage to the heart muscle resultant from a myocardial infarction (MI) triggers an immune response [13]. When this immune response is uncontrolled it can cause more damage to the heart. Phenolic compounds have been shown to modulate immune responses [14]; but there is a paucity of information on immunomodulatory effects of phenolic extracts from ginseng berry. Thus, our study also assessed immunomodulatory activity of GBE in the myocardial infarction (MI) model induced in rats by coronary artery ligation.

\section{Results}

\subsection{Phenolic Content and Antioxidant Capacity of GBE}

The total phenolic content of the GBE was $3586 \pm 04 \mathrm{mg}$ gallic acid equivalents $/ 100 \mathrm{~g}$ dry weight using the Folin-Ciocalteu assay. Oxygen radical absorbance capacity (ORAC) assay was performed to assess the oxygen radical scavenging activity of the extract. As expected, GBE exhibited a strong antioxidant capacity with a value of 151,864 $\pm 883 \mu \mathrm{mol}$ Trolox equivalents/100 g dry weight. The proximate analysis is presented in Table 1.

Table 1. Proximate analysis of ginseng berry extract.

\begin{tabular}{cc}
\hline Constituent & Value (\%) \\
\hline Moisture & 8.19 \\
Dry matter & 91.81 \\
Crude protein & 8.61 \\
Crude fibre & 1.54 \\
Fat & 0.90 \\
Ash & 12.50 \\
Non-fibre carbohydrates & 68.26 \\
Total digestible nutrients & 69.77 \\
\hline
\end{tabular}

\subsection{Body Weight and Heart Weight Characteristics after MI}

Biometrical characteristics were assessed to check whether the coronary artery ligation or treatment with GBE was able to produce any alterations. No significant changes in body weight 
and heart weight were observed between groups 8 weeks post-surgery. The percentage of scar weight was $21.31 \%$ less in GBE-treated MI animals, but not statistically significant when compared to the water-treated MI animals (Table 2). No differences were observed in the heart weight to tibia length ratio values between groups. Similarly, liver and lung weights also did not show significant differences between groups (values not shown).

Table 2. Biometrical characteristics of sham and MI animals with and without ginseng berry extract treatment for 8 weeks after coronary artery ligation.

\begin{tabular}{ccccc}
\hline Parameter & Sham-W & Sham-G & MI-W & MI-G \\
\hline Body weight $(\mathrm{g})$ & $573.1 \pm 11$ & $579.9 \pm 21$ & $538.4 \pm 12$ & $549 \pm 16$ \\
Heart weight $(\mathrm{g})$ & $1.433 \pm 0.05$ & $1.355 \pm 0.05$ & $1.458 \pm 0.03$ & $1.415 \pm 0.05$ \\
LV weight $(\mathrm{g})$ & $0.70 \pm 0.060$ & $0.86 \pm 0.051$ & $0.73 \pm 0.03$ & $0.82 \pm 0.04$ \\
\% infarct & - & - & $32.37 \pm 2.8$ & $25.47 \pm 2.3$ \\
Heart weight/Tibia length $(\mathrm{g} / \mathrm{cm})$ & $0.32 \pm 0.009$ & $0.30 \pm 0.01$ & $0.33 \pm 0.0$ & $0.31 \pm 0.0$ \\
LV weight/Tibia length $(\mathrm{g} / \mathrm{cm})$ & $0.15 \pm 0.013$ & $0.19 \pm 0.011$ & $0.16 \pm 0.01$ & $0.18 \pm 0.01$ \\
\hline
\end{tabular}

Values presented are mean \pm SEM. MI: Myocardial infarction; LV: Left Ventricle; Sham-W: Sham MI treated with distilled water $(n=8)$; Sham-G: Sham MI treated with GBE $150 \mathrm{mg} / \mathrm{kg} /$ body weight/day $(n=8)$; MI-W: MI treated with distilled water $(n=12-14)$; MI-G: MI treated with GBE $150 \mathrm{mg} / \mathrm{kg} /$ body weight/day $(n=12-14)$.

\subsection{Lack of Improvement in Cardiac Structure and Function with GBE Treatment}

M-mode echocardiography was carried out to assess the effect of GBE on the left ventricular remodeling at 4 and 8 weeks post-MI. At 4 weeks, left ventricle (LV) internal diameter (LVID) values at systole and diastole were comparable between water- and GBE-treated sham animals (Figure 1A,C). In contrast, the water-treated and GBE-treated MI groups had significantly higher LVID values when compared to the water-treated sham group indicating left ventricular dilatation (Figure $1 \mathrm{~A}, \mathrm{C}$ ). A similar trend was observed at 8 weeks with significantly high LVID values at systole and diastole in the water-treated and GBE-treated MI groups in comparison to water-treated sham group (Figure 1B,D). However, no changes were observed in LVID after GBE treatment in MI group (vs. water treated MI group) at 4 and 8 weeks (Figure 1A-D). LV volumes were also observed to be altered. MI animals treated with water and GBE showed significantly higher end-diastolic volume (EDV) and end-systolic volume (ESV) when compared to water treated sham animals (Figure 1E-H). There was no difference observed between sham animals treated with water and GBE, for EDV and ESV. GBE treatment of MI animals did not produce significant changes in EDV and ESV when compared to the water-treated MI animals (Figure 1E-H).

4 weeks

A

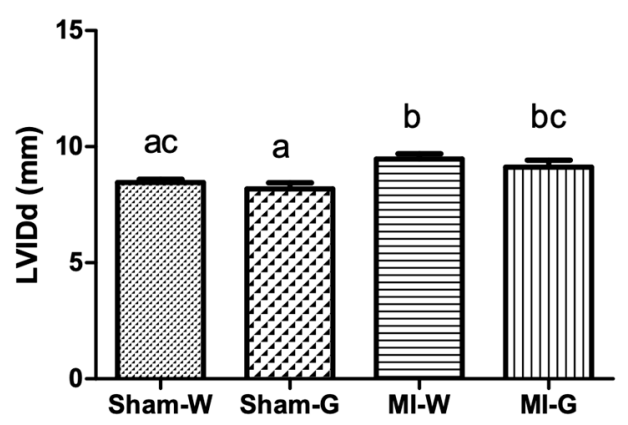

8 weeks

B

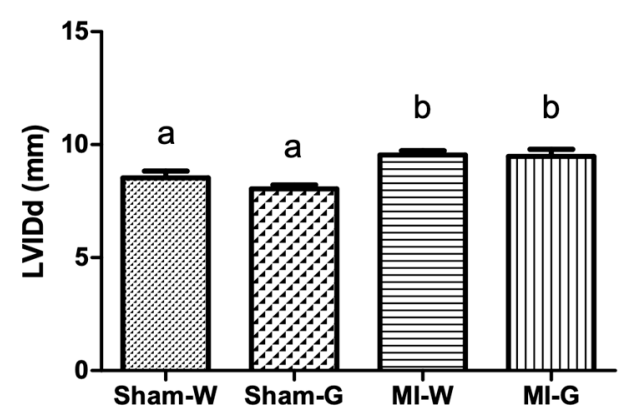

Figure 1. Cont. 
C

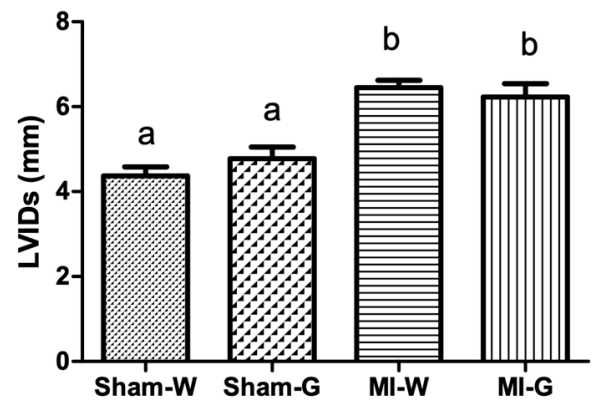

$\mathrm{E}$

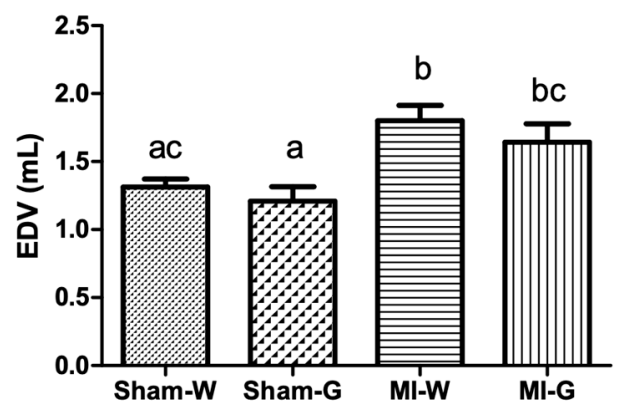

G

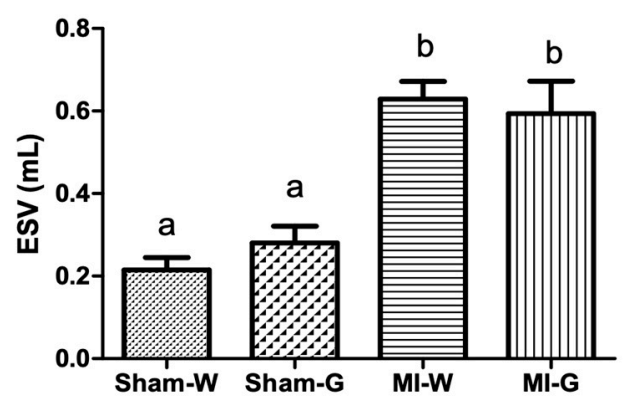

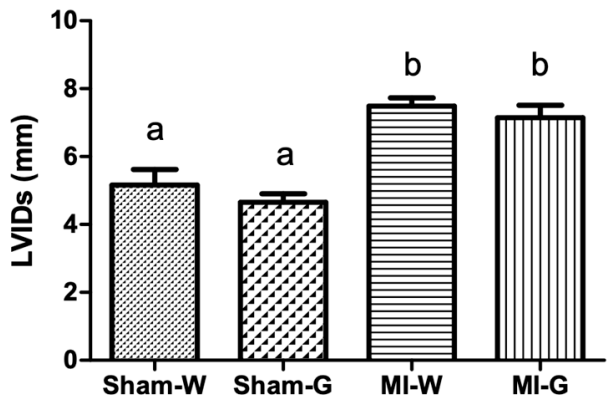

F

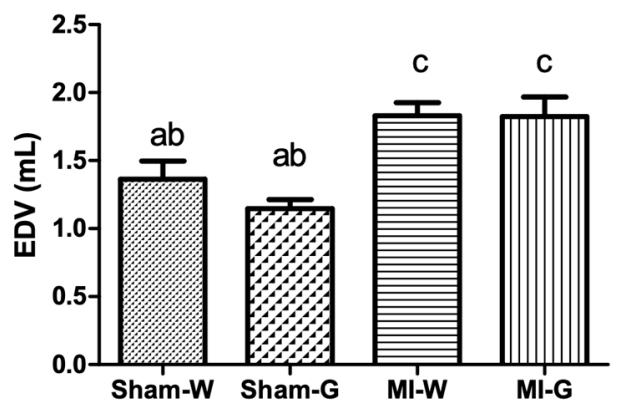

$\mathrm{H}$

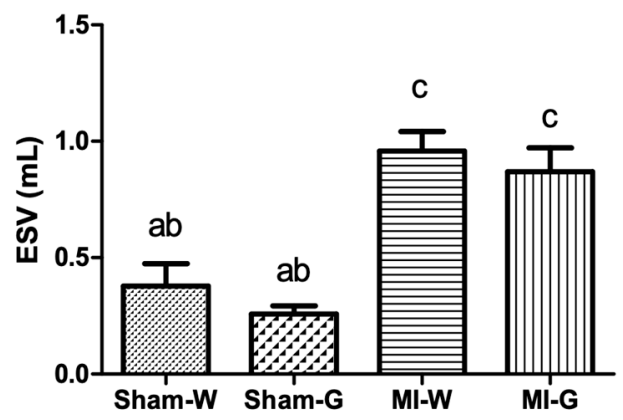

Figure 1. Effect of ginseng berry extract on ventricular chamber dilatation and remodeling. (A) LV internal diameter at diastole (LVIDd) at 4 weeks; (B) LVIDd at 8 weeks; (C) LV internal diameter at systole (LVIDs) at 4 weeks; (D) LVIDs at 8 weeks; (E) end diastolic volume (EDV) at 4 weeks; (F) EDV at 8 weeks; (G) end systolic volume (ESV) at 4 weeks; (H) ESV at 8 weeks. Values presented are mean \pm SEM. Bars with differing letter are significantly different, $p<0.05$. Sham-W: Sham MI treated with distilled water $(n=8)$; Sham-G: Sham MI treated with GBE $150 \mathrm{mg} / \mathrm{kg} /$ body weight $/$ day $(n=8)$; MI-W: MI treated with distilled water $(n=12-14)$; MI-G: MI treated with GBE $150 \mathrm{mg} / \mathrm{kg} / \mathrm{body}$ weight/day $(n=12-14)$.

To check for left ventricular hypertrophy along with the increased LV dilatation, the thickness of interventricular septal thickness (IVS) and LV posterior wall (LVPW) was measured. IVS thickness at systole was observed to be significantly lower at 4 weeks (Figure 2C), and at both systole and diastole at 8 weeks (Figure 2B,D), in the water- and GBE-treated MI animals when compared to water treated sham animals. LVPW thickness values were also found to be significantly low at systole in the water-treated MI group when compared to water treated sham group (Figure 2H). At both 4 and 8 weeks, values for IVS and LVPW thickness were found to be comparable between water and GBE treated MI groups (Figure 2A-H). 
4 weeks

A

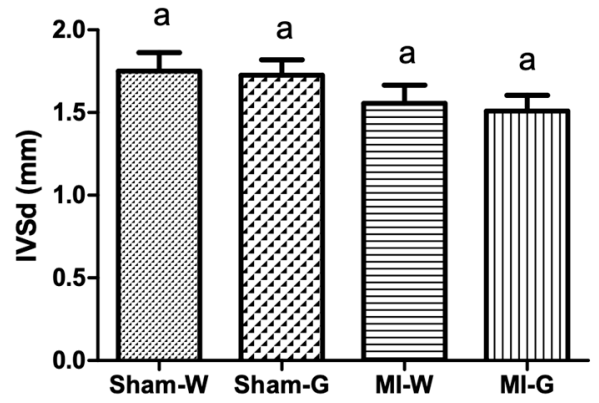

C

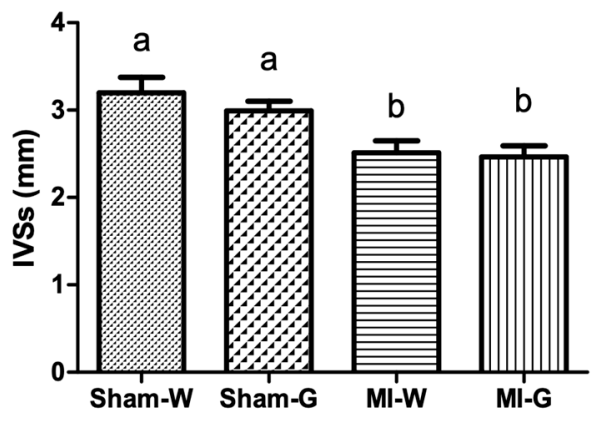

E

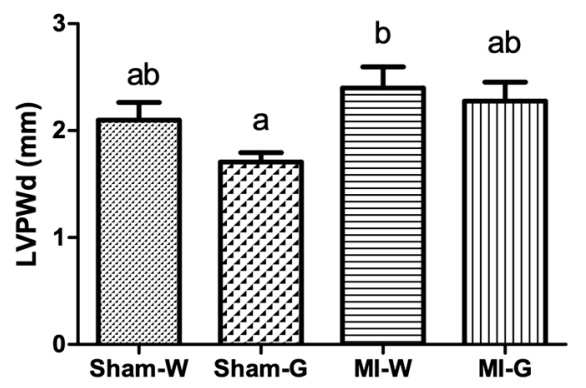

G

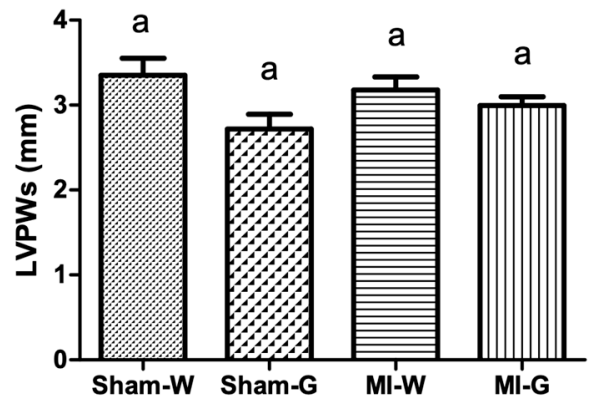

8 weeks

B

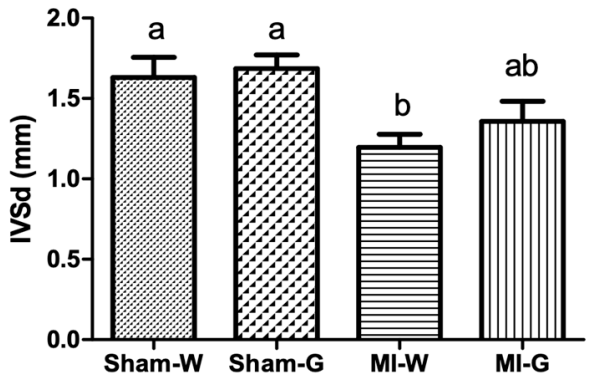

D

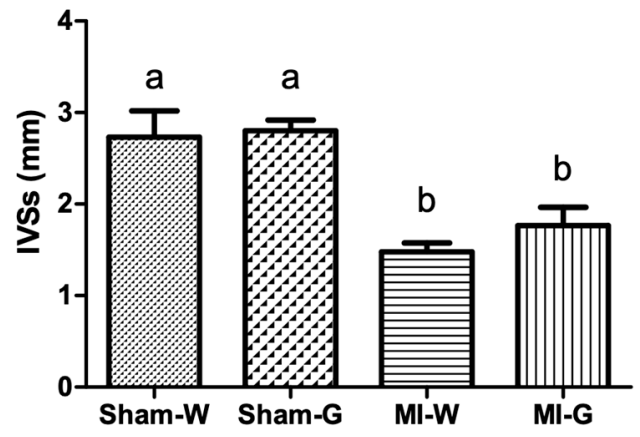

F

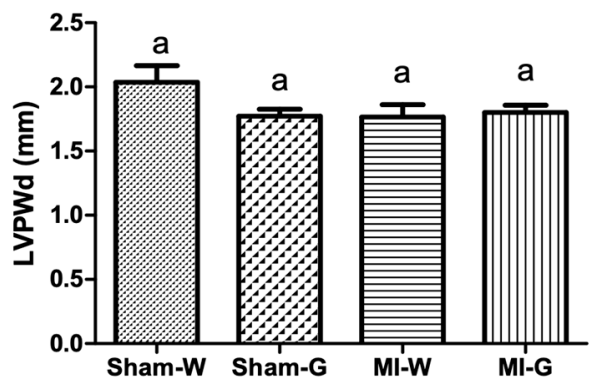

$\mathrm{H}$

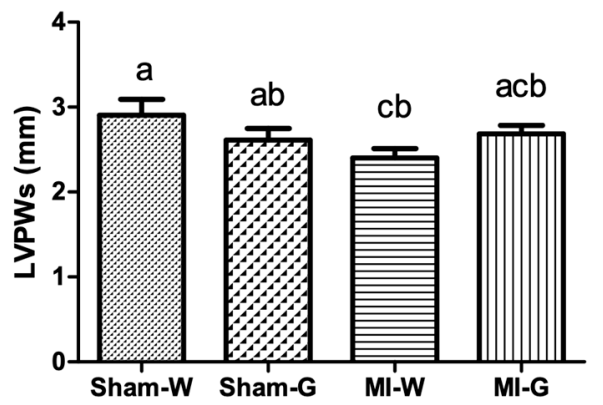

Figure 2. Effect of ginseng berry extract on cardiac hypertrophy. (A) Interventricular septal thickness at diastole (IVSd) at 4 weeks; (B) IVSd at 8 weeks; (C) IVS at systole (IVSs) at 4 weeks; (D) IVSs at 8 weeks; (E) LV posterior wall thickness at diastole (LVPWd) at 4 weeks; (F) LVPWd at 8 weeks; (G) LV posterior wall thickness at systole (LVPWs) at 4 weeks; (H) LVPWs at 8 weeks. Values presented are mean \pm SEM. Bars with differing letter are significantly different, $p<0.05$. Sham-W: Sham MI treated with distilled water $(n=8)$; Sham-G: Sham MI treated with GBE $150 \mathrm{mg} / \mathrm{kg} /$ body weight/day $(n=8) ; \mathrm{MI}-\mathrm{W}$ : MI treated with distilled water $(n=12-14)$; MI-G: MI treated with GBE $150 \mathrm{mg} / \mathrm{kg} /$ body weight/day $(n=12-14)$. 
Cardiac function was assessed by using M-mode echocardiography. Ejection fraction (EF), a measure of cardiac contraction (systolic heart function), was identical between water- and GBE-treated sham animals. The water-treated and GBE-treated MI groups showed significantly lower EF at 4 and 8 weeks when compared to water treated sham group (Figure 3A,B). EF values were not significantly different between the water- and GBE-treated MI groups (Figure 3A,B). Another parameter of cardiac contraction, fractional shortening (FS), was also significantly reduced in the water- and GBE-treated MI groups when compared to water treated Sham controls (Figure 3C,D). No significant differences were noted for FS values between water and GBE treated MI groups (Figure 3C,D).

4 weeks

A
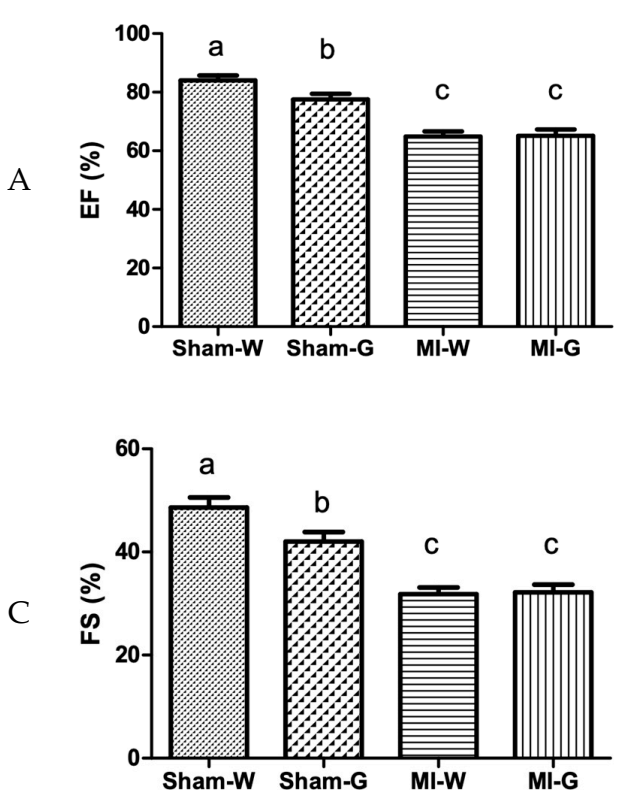

8 weeks
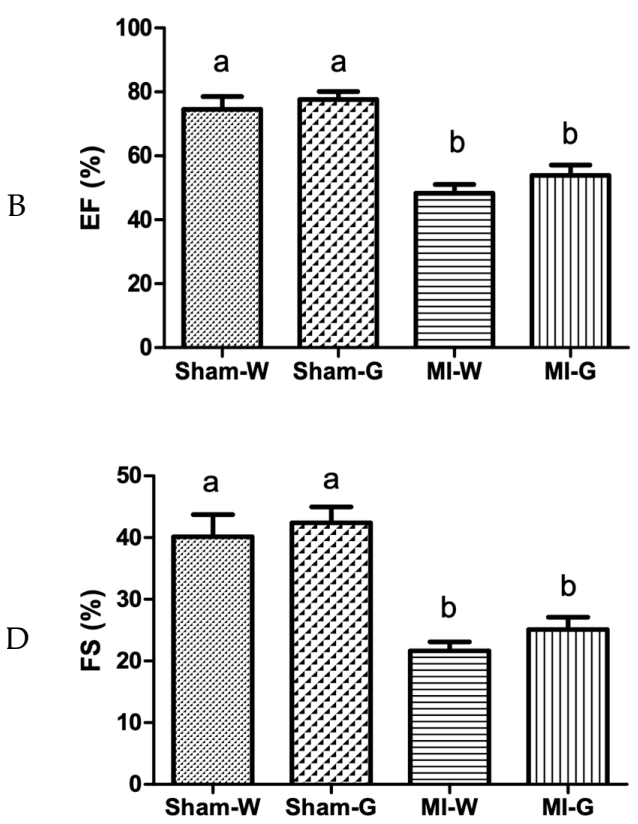

Figure 3. Effect of ginseng berry extract on systolic heart function. (A) Ejection fraction (EF) at 4 weeks; (B) EF at 8 weeks; (C) fractional shortening (FS) at 4 weeks; (D) FS at 8 weeks. Values presented are mean \pm SEM. Bars with differing letter are significantly different, $p<0.05$. Sham-W: Sham MI treated with distilled water $(n=8)$; Sham-G: Sham MI treated with GBE $150 \mathrm{mg} / \mathrm{kg} / \mathrm{body}$ weight/day $(n=8)$; MI-W: MI treated with distilled water $(n=12-14)$; MI-G: MI treated with GBE $150 \mathrm{mg} / \mathrm{kg} / \mathrm{body}$ weight/day $(n=12-14)$.

Doppler echocardiography was used to examine the changes in cardiac relaxation (diastolic heart function), post-MI. At 4 and 8 weeks, no changes were observed in the parameters assessing diastolic heart function, mitral valve (MV) $\mathrm{E}$ and A wave velocity in all groups (Figure 4A,B). At 4 weeks, the values of another diastolic heart function parameter, Isovolumic relaxation time (IVRT) was also found to be similar in all the groups (Figure 4C). However, IVRT was observed to be significantly increased in the water- and GBE-treated MI groups when compared to water treated sham controls at 8 weeks (Figure 4D). GBE-treated MI animals had values comparable to water-treated MI animals (Figure 4D).

\subsection{Reduction in Oxidative Stress and Inflammation with Ginseng Berry Extract Treatment}

The 8-week water treated MI animals demonstrated significantly increased MDA levels in the heart tissue when compared to water-treated sham animals (Figure 5A). The GBE treated MI group showed a significant decrease (29\%) in MDA levels when compared to the water-treated MI group (Figure 5A). Although, values for TNF- $\alpha$ were comparable between 8-week water treated sham and MI groups, a significant reduction of $\sim 94 \%$ was observed in 8-week GBE-treated MI and sham animals when compared to water-treated sham and MI animals, respectively (Figure 5B). 
4 weeks

A

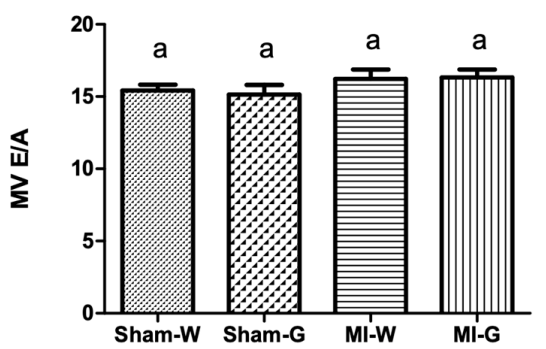

C

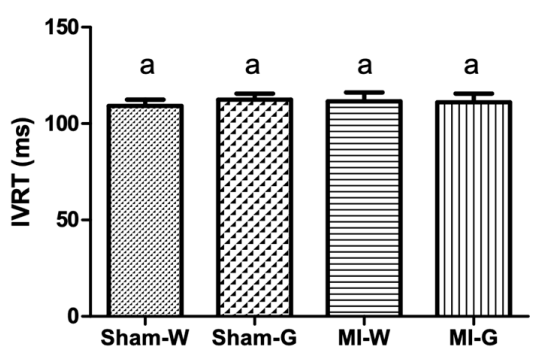

8 weeks

B

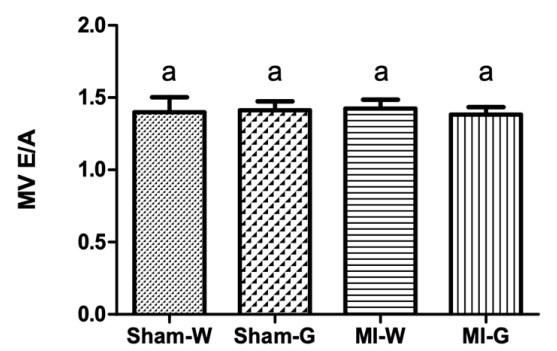

$\mathrm{D}$

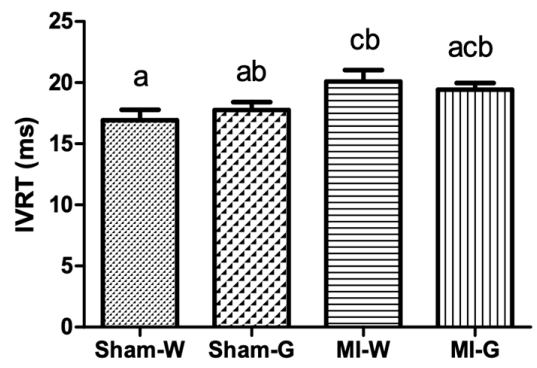

Figure 4. Effect of ginseng berry extract on diastolic heart function. (A) Mitral valve E- to A- wave ratio (MV E/A) at 4 weeks; (B) MV E/A at 8 weeks; (C) Isovolumic relaxation time (IVRT) at 4 weeks; (D) IVRT at 8 weeks. Values presented are mean \pm SEM. Bars with differing letter are significantly different, $p<0.05$. Sham-W: Sham MI treated with distilled water $(n=8)$; Sham-G: Sham MI treated with GBE $150 \mathrm{mg} / \mathrm{kg} /$ body weight/day $(n=8)$; MI-W: MI treated with distilled water $(n=12-14)$; MI-G: MI treated with GBE $150 \mathrm{mg} / \mathrm{kg} /$ body weight/day $(n=12-14)$.

A

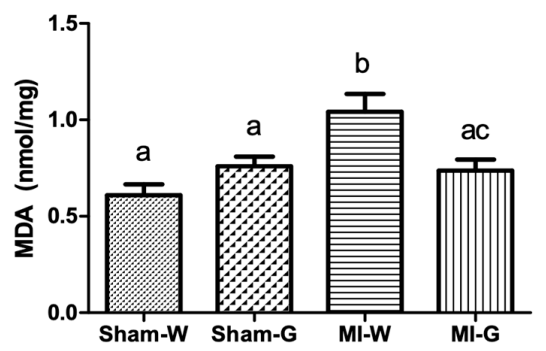

B

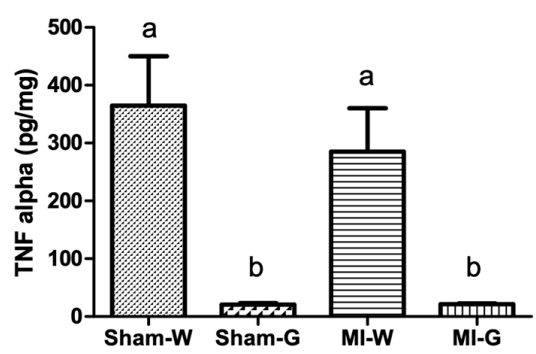

Figure 5. Effect of ginseng berry extract on oxidative stress and inflammation. (A) Malondialdehyde (MDA); (B) tumor necrosis factor- $\alpha$ (TNF- $\alpha)$. Values presented are mean \pm SEM. Bars with differing letter are significantly different, $p<0.05$. Sham-W: Sham MI treated with distilled water $(n=8)$; Sham-G: Sham MI treated with GBE $150 \mathrm{mg} / \mathrm{kg} /$ body weight/day $(n=8) ;$ MI-W: MI treated with distilled water ( $n=12-14)$; MI-G: MI treated with GBE $150 \mathrm{mg} / \mathrm{kg} /$ body weight/day $(n=12-14)$.

\subsection{Immune Cell Phenotypes}

Using forward vs. side scatter we can identify viable lymphoid cells. Cell shrinkage, one of the first indicators of apoptosis, can be identified by a decrease in forward light scatter. There was no difference among groups in the proportion of cells that appeared in the non-viable lymphoid region.

In the viable lymphoid region (Figure 6), MI had no effect on the proportion of total T-cells (identified as $\mathrm{CD}^{+}$), helper T-cells (identified as $\mathrm{CD}^{+} \mathrm{CD}^{+} \mathrm{CD} 8^{-}$), cytotoxic T-cells (identified as $\mathrm{CD}^{+} \mathrm{CD}^{-} \mathrm{CD}^{+}$) or activated T-cells (identified as $\mathrm{CD}^{+} \mathrm{CD} 25^{+}$) and activated cytotoxic T-cells (identified as $\mathrm{CD}^{+} \mathrm{CD} 4^{-} \mathrm{CD} 8^{+} \mathrm{CD} 25^{+}$). MI did result in an $8.0 \%$ lower proportion of activated helper T-cells (identified as $\mathrm{CD}^{+} \mathrm{CD}^{+} \mathrm{CD}^{-} \mathrm{CD}^{-} 5^{+}$) and $9.8 \%$ lower proportion of T-regulatory cells (identified as $\mathrm{CD} 3^{+} \mathrm{CD} 4^{+} \mathrm{CD} 8^{-} \mathrm{CD} 25^{+}$foxp $3^{+}$) compared to sham-operated rats. GBE treated rats had a $9.4 \%$ higher proportion of T-cells and 13.2\% higher proportion of cytotoxic T-cells, but a $7.4 \%$ lower proportion of helper T-cells compared to water treated rats. There was no effect of GBE on the 
proportion of activated T-cells, activated helper T-cells, activated cytotoxic T-cells and T-regulatory cells (Table 3).

A

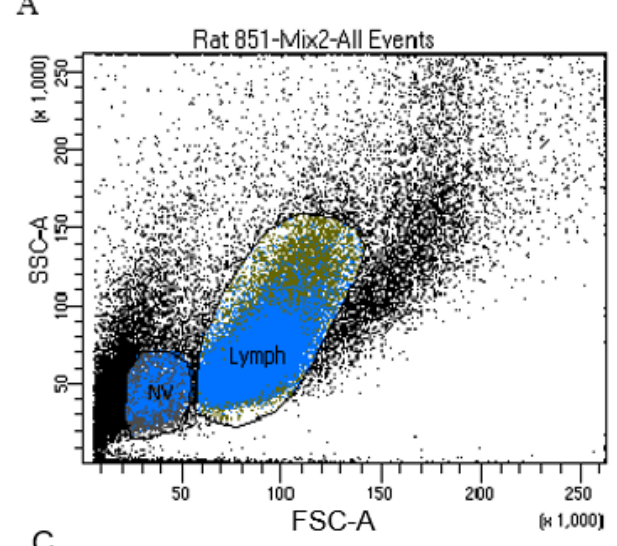

C

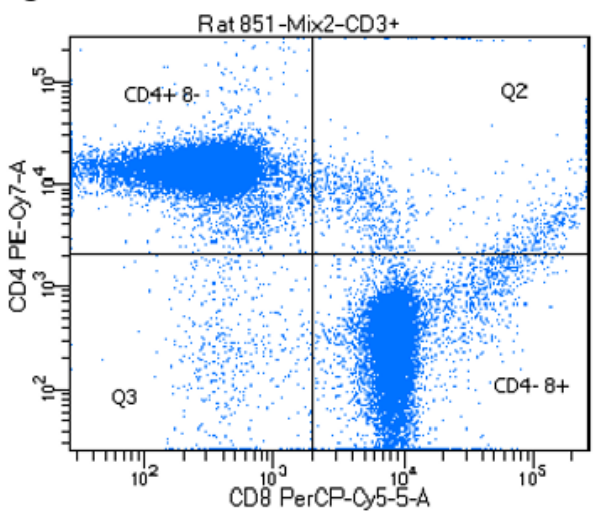

E

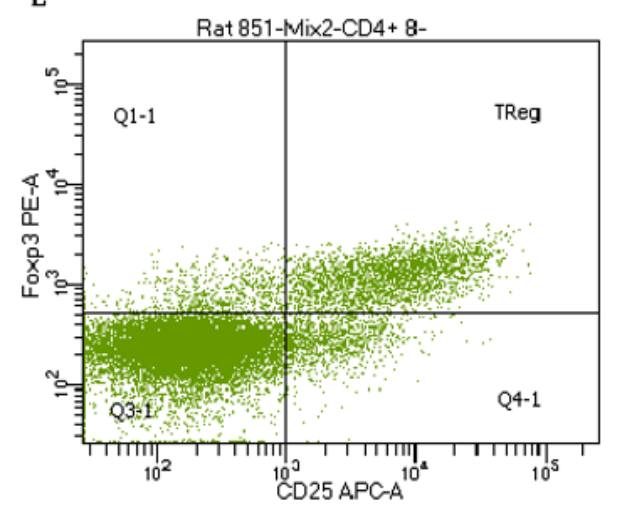

B

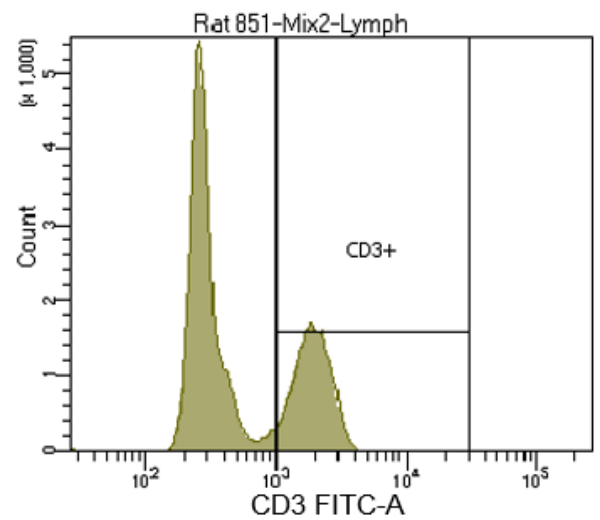

D

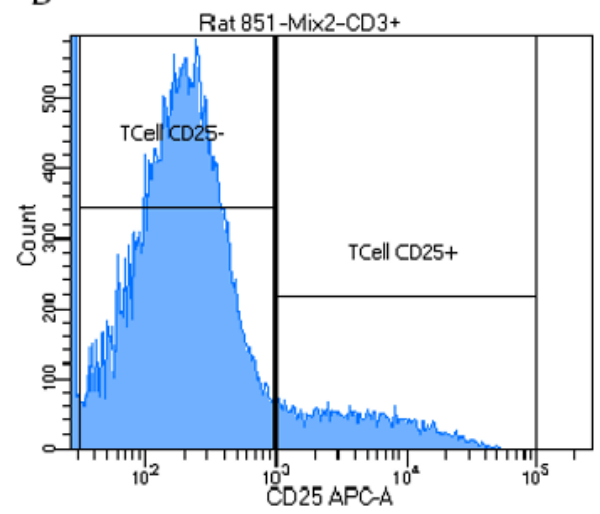

F

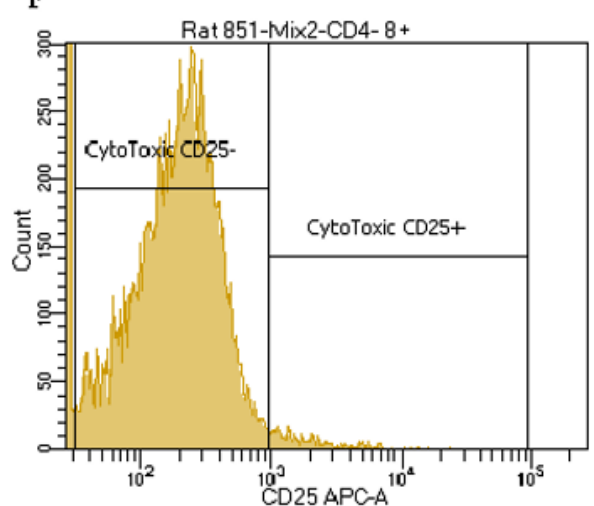

Figure 6. Immune cell phenotyping. (A) Definition of viable lymphocytes (Lymph) and non-viable lymphocytes (NV); (B) Definition of CD3 binding after gating on Lymph; (C) Definition of CD4 and CD8 after gating on $\mathrm{CD}^{+}$Lymph; (D) Definition of CD25 binding after gating on $\mathrm{CD}^{+}$Lymph; (E) Definition of Foxp3 and CD25 after gating on $\mathrm{CD}^{+} \mathrm{CD}^{+}{ }^{+} \mathrm{CD} 8^{-}$Lymph; (F) Definition of CD25 binding after gating on $\mathrm{CD}^{+} \mathrm{CD} 4^{-} \mathrm{CD} 8^{+}$Lymph.

In the non-viable lymphoid region compared to the viable lymphoid region, there were lower proportions of all T-cell phenotypes, except for helper T-cells (Figure 7). Rats that had an MI had a $30 \%$ lower proportion of activated cytotoxic T-cells compared to sham-operated rats in the non-viable region. There was a trend towards a lower proportion of T-regulatory cells after MI compared to sham $(p=0.06)$, but no significant difference in the proportion of total T-cells, activated T-cells, helper T-cells, activated helper T-cells, or cytotoxic T-cells in the non-viable region. Compared to water-treated controls, rats treated with GBE had a $29.4 \%$ higher proportion of T-cells and a $10 \%$ lower proportion of 
activated Helper T-cells in this region. There was no difference in the proportion of activated T-cells, helper T-cells, T-regulatory cells, cytotoxic T-cells and activated cytotoxic T-cells (Table 3).

A

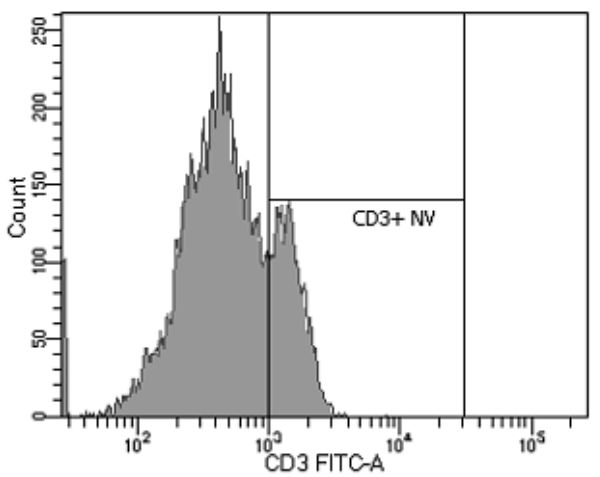

B

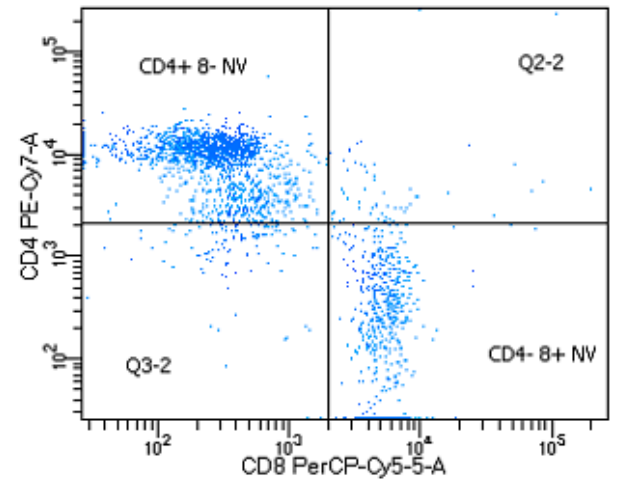

$\mathrm{D}$

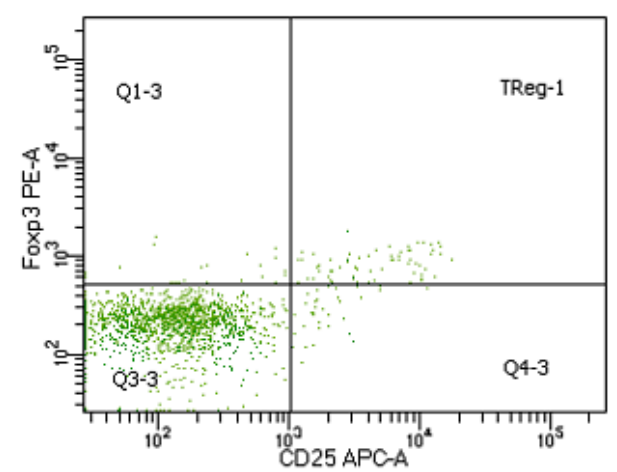

$\mathrm{C}$

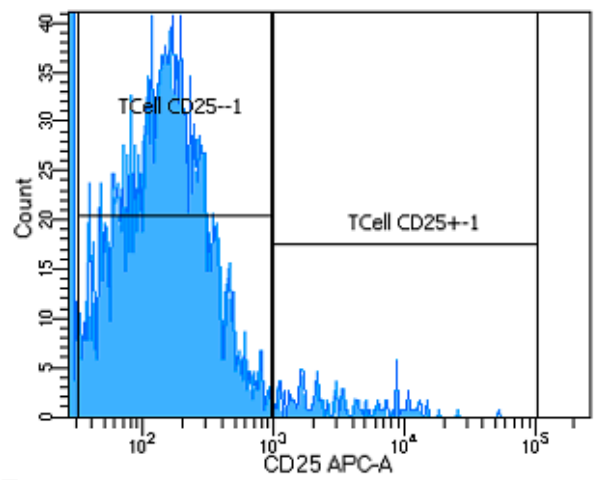

E

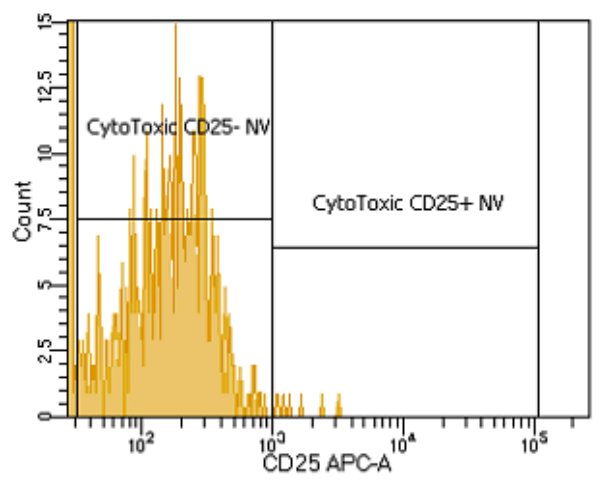

Figure 7. Representative flow cytometry plot for non-viable lymphocytes. (A) Definition of CD3 binding after gating on non-viable lymphocytes (NV); (B) Definition of CD4 and CD8 after gating on $\mathrm{CD}^{+} \mathrm{NV}$; (C) Definition of CD25 binding after gating on $\mathrm{CD}^{+} \mathrm{NV}$; (D) Definition of Foxp3 and CD25 after gating on $\mathrm{CD}^{+} \mathrm{CD}^{+} \mathrm{CD}^{-} \mathrm{NV}$; (E) Definition of $\mathrm{CD} 25$ binding after gating on $\mathrm{CD}^{+} \mathrm{CD} 4^{-} \mathrm{CD}^{+} \mathrm{NV}$.

\subsection{Cytokines Production}

ConA stimulated splenocytes from rats that had an MI when compared to controls, did not show a significant difference in IL-2, IFN $\gamma$, IL-10 or TNF $\alpha$ concentrations produced or between GBE treatment and control (Table 4).

Unstimulated samples were below the lower limit of detection for IFN $\gamma(6.8 \mathrm{pg} / \mathrm{mL})$ and TNF $\alpha$ $(27.7 \mathrm{pg} / \mathrm{mL})$. Unstimulated concentrations of IL-2 and IL-10 were not affected by MI or GBE treatment (Table 4). 
Table 3. T-cell phenotypes in viable and non-viable gates.

\begin{tabular}{|c|c|c|c|c|c|c|c|c|c|c|c|}
\hline \multirow[b]{2}{*}{$\begin{array}{l}\text { Lymphocyte } \\
\text { Phenotypes }\end{array}$} & \multicolumn{3}{|c|}{ Model } & \multicolumn{3}{|c|}{ Treatment } & \multicolumn{5}{|c|}{ Interaction } \\
\hline & $\begin{array}{l}\text { Sham } \\
n=16\end{array}$ & $\begin{array}{c}\text { MI } \\
n=23\end{array}$ & $p$-Value & $\begin{array}{l}\text { Water } \\
n=20\end{array}$ & $\begin{array}{c}\text { GBE } \\
n=19\end{array}$ & $p$-Value & $\begin{array}{c}\text { Sham Water } \\
n=8\end{array}$ & $\begin{array}{c}\text { Sham GBE } \\
n=8\end{array}$ & $\begin{array}{c}\text { MI Water } \\
n=12\end{array}$ & $\begin{array}{c}\text { MI GBE } \\
n=11\end{array}$ & $p$-Value \\
\hline \multicolumn{12}{|l|}{ Viable Lymphocytes } \\
\hline $\begin{array}{c}\mathrm{CD}^{+} \\
\text {\% lymphocytes }\end{array}$ & $30.0 \pm 0.9$ & $30.0 \pm 0.8$ & 0.99 & $28.7 \pm 0.8$ & $31.4 \pm 0.9$ & 0.03 & $29.2 \pm 1.3^{a b}$ & $30.9 \pm 1.3^{a b}$ & $28.2 \pm 1.0^{\mathrm{b}}$ & $31.9 \pm 1.1^{\mathrm{a}}$ & 0.39 \\
\hline $\begin{array}{l}{ }^{1} \mathrm{CD}_{25}^{+} \\
\% \text { of } \mathrm{CD}^{+}\end{array}$ & $11.5 \pm 0.3$ & $10.9 \pm 0.3$ & 0.14 & $11.5 \pm 0.3$ & $10.9 \pm 0.3$ & 0.15 & $11.7 \pm 0.5$ & $11.3 \pm 0.4$ & $11.3 \pm 0.4$ & $10.4 \pm 0.4$ & 0.65 \\
\hline $\begin{array}{l}\mathrm{CD}^{+} \mathrm{CD}^{-} \\
\% \text { of } \mathrm{CD}^{+}\end{array}$ & $57.1 \pm 1.2$ & $57.5 \pm 1.0$ & 0.80 & $59.5 \pm 1.1$ & $55.1 \pm 1.1$ & 0.01 & $58.7 \pm 1.7^{\mathrm{a}}$ & $55.5 \pm 1.7^{\mathrm{ab}}$ & $60.4 \pm 1.4^{\mathrm{a}}$ & $54.6 \pm 1.5^{b}$ & 0.41 \\
\hline $\begin{array}{c}{ }^{2} \mathrm{CD}_{25^{+}} \\
\% \text { of } \mathrm{CD} 3^{+} \mathrm{CD} 4^{+} \mathrm{CD} 8^{-}\end{array}$ & $16.3 \pm 0.4$ & $15.0 \pm 0.4$ & 0.04 & $15.9 \pm 0.4$ & $15.4 \pm 0.4$ & 0.40 & $16.7 \pm 0.6^{\mathrm{a}}$ & $15.9 \pm 0.6^{\mathrm{ab}}$ & $15.1 \pm 0.6^{\mathrm{ab}}$ & $14.9 \pm 0.6^{\mathrm{b}}$ & 0.54 \\
\hline $\begin{array}{c}{ }^{3} \mathrm{Foxp}^{++} \mathrm{CD} 25^{+} \\
\% \text { of } \mathrm{CD}^{+} \mathrm{CD} 4^{+} \mathrm{CD} 8^{-}\end{array}$ & $12.3 \pm 0.4$ & $11.1 \pm 0.3$ & 0.03 & $11.9 \pm 0.4$ & $11.6 \pm 0.4$ & 0.60 & $12.8 \pm 0.5^{\mathrm{a}}$ & $11.8 \pm 0.5^{\mathrm{ab}}$ & $10.9 \pm 0.5^{b}$ & $11.4 \pm 0.5^{\mathrm{ab}}$ & 0.17 \\
\hline $\begin{array}{l}\mathrm{CD} 4^{-} \mathrm{CD}^{+} \\
\% \text { of } \mathrm{CD}^{+} \\
\end{array}$ & $39.6 \pm 1.2$ & $39.2 \pm 1.0$ & 0.80 & $37.0 \pm 1.1$ & $41.9 \pm 1.1$ & 0.004 & $37.8 \pm 1.7^{a b}$ & $41.5 \pm 1.7^{\mathrm{a}}$ & $36.2 \pm 1.4^{\mathrm{b}}$ & $42.3 \pm 1.5^{\mathrm{a}}$ & 0.46 \\
\hline $\begin{array}{c}{ }^{4} \mathrm{CD}^{\mathrm{C}} 5^{+} \\
\% \text { of } \mathrm{CD} 3^{+} \mathrm{CD} 4^{-} \mathrm{CD} 8^{+}\end{array}$ & $2.8 \pm 0.2$ & $2.7 \pm 0.1$ & 0.66 & $2.7 \pm 0.2$ & $2.7 \pm 0.1$ & 0.97 & $2.7 \pm 0.2$ & $2.9 \pm 0.2$ & $2.8 \pm 0.2$ & $2.6 \pm 0.2$ & 0.30 \\
\hline \multicolumn{12}{|l|}{ Non-Viable lymphocytes } \\
\hline $\begin{array}{c}\mathrm{CD}^{+} \\
\% \text { non-viable cells }\end{array}$ & $27.2 \pm 1.1$ & $25.7 \pm 0.9$ & 0.31 & $23.1 \pm 1.0$ & $29.9 \pm 1.0$ & $<0.0001$ & $22.9 \pm 1.5^{b}$ & $31.5 \pm 1.5^{\mathrm{a}}$ & $23.2 \pm 1.3^{b}$ & $28.2 \pm 1.4^{\mathrm{a}}$ & 0.22 \\
\hline $\begin{array}{c}{ }^{5} \mathrm{CD}_{2} 5^{+} \\
\% \text { of } \mathrm{CD}^{+}\end{array}$ & $4.9 \pm 0.2$ & $4.3 \pm 0.2$ & 0.07 & $4.9 \pm 0.2$ & $4.3 \pm 0.2$ & 0.06 & $4.9 \pm 0.3^{\mathrm{a}}$ & $4.8 \pm 0.3^{\mathrm{a}}$ & $4.8 \pm 0.3^{\mathrm{a}}$ & $3.9 \pm 0.3^{b}$ & 0.17 \\
\hline $\begin{array}{l}\mathrm{CD} 4^{+} \mathrm{CD}^{-} \\
\% \text { of } \mathrm{CD}^{+}\end{array}$ & $75.6 \pm 1.3$ & $74.4 \pm 1.1$ & 0.49 & $74.9 \pm 1.1$ & $75.1 \pm 1.2$ & 0.94 & $75.7 \pm 1.8$ & $75.5 \pm 1.8$ & $74.2 \pm 1.5$ & $74.7 \pm 1.6$ & 0.83 \\
\hline $\begin{array}{c}{ }^{6} \mathrm{CD}_{25^{+}} \\
\% \text { of } \mathrm{CD} 3^{+} \mathrm{CD} 4^{+} \mathrm{CD} 8^{-}\end{array}$ & $4.9 \pm 0.2$ & $4.5 \pm 0.2$ & 0.13 & $4.9 \pm 0.2$ & $4.4 \pm 0.2$ & 0.05 & $4.9 \pm 0.3^{\mathrm{a}}$ & $4.9 \pm 0.3^{\mathrm{a}}$ & $5.0 \pm 0.2^{\mathrm{a}}$ & $4.0 \pm 0.2^{b}$ & 0.08 \\
\hline $\begin{array}{c}{ }^{{ }^{7} \mathrm{Foxp}^{+} \mathrm{CD} 25^{+}} \\
\% \text { of } \mathrm{CD}^{+} \mathrm{CD} 4^{+} \mathrm{CD} 8^{-}\end{array}$ & $3.1 \pm 0.1$ & $2.7 \pm 0.1$ & 0.06 & $3.0 \pm 0.1$ & $2.8 \pm 0.1$ & 0.18 & $3.2 \pm 0.2^{\mathrm{a}}$ & $3.0 \pm 0.2^{\mathrm{ab}}$ & $2.9 \pm 0.2^{\mathrm{ab}}$ & $2.6 \pm 0.2^{b}$ & 0.59 \\
\hline $\begin{array}{l}\mathrm{CD} 4^{-} \mathrm{CD} 8^{+} \\
\% \text { of } \mathrm{CD} 3+\end{array}$ & $17.5 \pm 0.9$ & $18.3 \pm 0.8$ & 0.56 & $17.3 \pm 0.9$ & $18.5 \pm 0.9$ & 0.36 & $16.7 \pm 1.3$ & $18.4 \pm 1.3$ & $18.0 \pm 1.1$ & $18.6 \pm 1.2$ & 0.66 \\
\hline $\begin{array}{c}{ }^{8} \mathrm{CD} 25^{+} \\
\% \text { of } \mathrm{CD}^{+} \mathrm{CD} 4^{-} \mathrm{CD} 8^{+}\end{array}$ & $2.3 \pm 0.3$ & $1.6 \pm 0.2$ & 0.05 & $2.2 \pm 0.2$ & $1.7 \pm 0.2$ & 0.17 & $2.4 \pm 0.4^{\mathrm{a}}$ & $2.2 \pm 0.4^{\mathrm{ab}}$ & $1.9 \pm 0.3^{\mathrm{ab}}$ & $1.2 \pm 0.3^{\mathrm{b}}$ & 0.54 \\
\hline
\end{tabular}

Values are means $(\mathrm{pg} / \mathrm{mL}) \pm$ standard error. Means with different superscript letters are significantly different $(p<0.05)$. GBE: Ginseng berry extract. ${ }^{1,4}$ : Outliers (value $>3 \times$ standard deviation of mean) were removed: 2 from MI water and 1 from sham water group; ${ }^{2,3,7}$ : Outliers (value $>3 \times$ standard deviation of mean) were removed: 2 from MI water; ${ }^{5,6,8}$ : Outlier (value $>3 \times$ standard deviation of mean) were removed: 1 from MI water. 
Table 4. Supernatant cytokine concentrations from concanvalin A-stimulated and unstimulated splenocytes.

\begin{tabular}{|c|c|c|c|c|c|c|c|c|c|c|c|}
\hline \multirow[b]{2}{*}{ Cytokines } & \multicolumn{3}{|c|}{ Model } & \multicolumn{3}{|c|}{ Treatment } & \multicolumn{5}{|c|}{ Interaction } \\
\hline & $\begin{array}{l}\text { Sham } \\
n=16\end{array}$ & $\begin{array}{c}\text { MI } \\
n=23\end{array}$ & $p$-Value & $\begin{array}{l}\text { Water } \\
n=20\end{array}$ & $\begin{array}{c}\text { GBE } \\
n=19\end{array}$ & $p$-Value & $\begin{array}{c}\text { Sham Water } \\
\quad n=8\end{array}$ & $\begin{array}{l}\text { Sham GBE } \\
\quad n=8\end{array}$ & $\begin{array}{c}\text { MI Water } \\
n=12\end{array}$ & $\begin{array}{c}\text { MI GBE } \\
n=11\end{array}$ & $p$-Value \\
\hline IL-2 ConA & $2111 \pm 181$ & $1890 \pm 79$ & 0.24 & $1910 \pm 133$ & $2055 \pm 114$ & 0.47 & $2067 \pm 279$ & $2155 \pm 248$ & $1805 \pm 125$ & $1983 \pm 90$ & 0.80 \\
\hline${ }^{1}$ IL-2 UNS & $3.4 \pm 0.2$ & $4.0 \pm 0.4$ & 0.35 & $3.9 \pm 0.4$ & $3.4 \pm 0.3$ & 0.52 & $3.5 \pm 0.6$ & $3.3 \pm 0.2$ & $4.2 \pm 0.6$ & $3.6 \pm 0.5$ & 0.72 \\
\hline${ }^{2}$ IFN $\gamma$ ConA & $383.0 \pm 75$ & $267.0 \pm 35$ & 0.13 & $312.9 \pm 59$ & $312.7 \pm 47$ & 0.79 & $427.8 \pm 131$ & $343.7 \pm 89$ & $245.9 \pm 49$ & $290.1 \pm 51$ & 0.40 \\
\hline IFN $\gamma$ UNS & ND & ND & I & ND & ND & 1 & ND & ND & ND & ND & I \\
\hline${ }^{3}$ IL-10 ConA & $49.2 \pm 3.3$ & $47.3 \pm 3.1$ & 0.65 & $48.4 \pm 4.1$ & $47.9 \pm 2.3$ & 0.81 & $51.9 \pm 5.4$ & $46.9 \pm 4.2$ & $45.9 \pm 5.9$ & $48.7 \pm 2.7$ & 0.41 \\
\hline${ }^{4}$ IL-10 UNS & $33.1 \pm 4.2$ & $29.1 \pm 1.8$ & 0.49 & $28.3 \pm 2.4$ & $33.1 \pm 3.1$ & 0.30 & $29.2 \pm 6.8$ & $35.4 \pm 5.6$ & $27.9 \pm 2.4$ & $30.8 \pm 2.8$ & 0.70 \\
\hline $\mathrm{TNF} \alpha \mathrm{ConA}$ & $773.6 \pm 57$ & $727.4 \pm 31$ & 0.43 & $779.1 \pm 42$ & $711.8 \pm 41$ & 0.30 & $792.9 \pm 87$ & $754.3 \pm 79$ & $770.0 \pm 42$ & $680.9 \pm 43$ & 0.68 \\
\hline TNF $\alpha$ UNS & ND & ND & / & ND & ND & / & ND & ND & ND & ND & / \\
\hline
\end{tabular}

Values are means $(\mathrm{pg} / \mathrm{mL}) \pm$ standard error. Abbreviations: Ginseng berry extract (GBE); Interleukin-2 (IL-2); concanvalin A (ConA); unstimulated (UNS); interferon- $\gamma$ (IFN $\gamma$ ); interleukin-10 (IL-10); tumor necrosis factor $\alpha$ (TNF $\alpha$ ); ND = not detectable. ${ }^{1}$ IL-2 UNS 4 samples from sham/water, 2 from sham/GBE, 4 from MI/water, 6 from MI/GBE were below detection limit of $0.46 \mathrm{pg} / \mathrm{Ml} ;{ }^{2}$ IFN $\gamma$ ConA 1 outlier removed from sham/water group; ${ }^{3}$ IL-10 ConA 2 samples from MI/water and 1 from sham/water were below detection limit of $19.4 \mathrm{pg} / \mathrm{mL} ;{ }^{4}$ IL-10 UNS 5 samples from sham/water, 3 from sham/GBE, 5 from MI/water, 6 from MI/GBE were below detection limit of $19.4 \mathrm{pg} / \mathrm{mL}$. 


\section{Discussion}

The present study is the first to report that ginseng berry phenolic extract has a strong antioxidant effect despite no effect on cardiac structure and function in the condition of a MI.

Although there is no information in the literature on the effects of ginseng berry on heart structure and function in any settings of cardiovascular disease, some studies conducted with Korean ginseng (Panax ginseng) have shown that GBE exerts anti-atherosclerotic [15], anti-diabetic, and anti-obesity effects [16]. Unlike ginseng root extract, GBE has been found to inhibit the mRNA expression of interleukins $1 \beta$ and 6 inflammatory markers and it is likely through this mechanism that it exerts its antihyperglycemic and anti-obesity effects [17]. GBE also increases prothrombin time, indicating potential atherothrombotic effects [8].

Ginseng consists of phenolic compounds which include phenolic acids and flavonoids with strong antioxidant activity, but these are less characterized as compared to the ginsenosides. Chung et al. reported 4-9-fold higher phenolic compounds in the 3-6 year-old Korean ginseng berry (Panax ginseng) compared to the same age roots [3]. A higher phenolic content has been observed in the older Korean ginseng berries when compared to younger ones; the total amount increasing by $20-48 \%$ with an increase in the cultivation year. DPPH free-radical-scavenging activity (DPPH activity) of the 3-6 years old Korean ginseng berry is 3-5 fold higher than the root, suggesting the strong antioxidant activity of the berry [3]. In this context, an extract derived from American ginseng berries protected cardiomyocytes from oxidative stress induced by $\mathrm{H}_{2} \mathrm{O}_{2}$ and antimycin A [8]. An approximately $60 \%$ reduction in cardiomyocyte death was observed with the American GBE, clearly demonstrating that it can salvage cardiomyocytes from the oxidative injury [18]. The proposed mechanisms for the American GBE antioxidant actions included direct free radical scavenging activity and stimulation of NO synthesis [18]. This study, therefore, examined the antioxidant potential of North American GBE (Panax quinquefolius) in addition to examining its cardioprotective potential in an experimental model of MI.

The present study demonstrated a significant reduction in ventricular function after a MI. The reasons behind this adverse outcome include an impaired contractile function as well as infarct expansion leading to ventricular dilatation. Two-dimensional echocardiography performed on patients with an acute transmural MI has demonstrated dilatation of the infarct zone. This infarct expansion phase, which occurs due to the slippage of necrotic myocardial fibers, starts as early as 3 days after infarction [19]. This early regional dilation occurring in the infarcted zone results in an overall left ventricular dilatation. The ventricular dilatation may continue until 30 months after infarction. Unlike the early expansion phase, both infarcted and non-infarcted segments are affected during the chronic phase dilation [20]. This disproportionate cardiac dilatation alters left ventricular topography and is associated with poor prognosis for long-term survival of MI patients [19]. Left ventricular dilatation has been associated with the progression of cardiac dysfunction as cardiac and stroke indices decrease. Of note, ventricular dilatation results in and is not the result of deteriorating cardiac function [21]. Left ventricular function is usually assessed by ejection fraction and end diastolic and systolic volumes. Ejection fraction has been observed to have a strong effect on mortality. With the increase in left ventricular internal diameter, there are substantial increases in end systolic and diastolic volumes with consequently increased stress on ventricular wall. Earlier studies with gated scintiphotography revealed an increase in left ventricular end-systolic volume and decrease in ejection fraction in MI patients compared to normal subjects [22]. Three months follow-up demonstrated increased values of LV ejection fraction in patients with clinical improvement and decreased values with clinically worsened cases [22]. Moller et al demonstrated that echocardiographically determined ejection fraction was a powerful predictor of mortality during a median follow up of 19 months [23]. A progressive increase was noted in the cardiac mortality in patients with ejection fraction below $40 \%$ [24]. Another important determinant of the ventricular function post-MI is the end systolic volume (ESV). Consideration of ESV along with EF adds more predictive power for mortality risk 
stratification of MI patients [25]. Echocardiographic analysis revealed that GBE treatment does not prevent infarct-related impairment in cardiac structure and function.

Reactive oxygen species produced during oxidative stress damage membrane lipids, proteins, DNA thereby causing apoptosis of cardiomyocytes and eventually resulting in cardiac dysfunction [26]. Infiltration of inflammatory cells in the myocardium after an ischemic event initiates an exaggerated inflammatory response, this further accelerates and worsens ventricular remodeling by increasing myocardial injury [27]. Higher levels of the inflammatory marker TNF- $\alpha$ have been associated with ventricular dilation and cardiac fibrosis. Our results demonstrate a significant decrease in the LV levels of TNF- $\alpha$ and MDA due to GBE treatment, indicating that it can reduce the inflammatory and oxidative response in MI. The substantial attenuation of cardiac oxidative stress observed in MI rats could be associated with the robust antioxidant capacity of GBE, as determined by its oxygen radical scavenging activity. Despite exerting strong antioxidant effects, however, GBE was unable to ameliorate cardiac remodeling and rescue cardiac function in MI rats.

The large GBE mediated decline in cardiac inflammatory response observed in the present study is consistent with a previous report which showed that treatment with Korean ginseng berry (Panax ginseng) suppressed the expression of TNF- $\alpha$ and thereby reduced atherosclerotic lesions [15]. Chronic use of TNF- $\alpha$ blockers has nevertheless been associated with increased risk of cardiovascular complications. A deficiency in TNF signaling for an extended period of time can cause immune system defects. The cardiac effects of TNF- $\alpha$ are biphasic. While high levels of TNF- $\alpha$ is associated with apoptosis, the basal level of TNF- $\alpha$ is required to maintain cytoprotective Nrf2 signaling [28]. Kurrelmeyer et al reported the importance of TNF signaling in protecting cardiomyocytes against ischemic injury [29]. The protective mechanism against hypoxic damage could be via activation of protein kinase A which stimulates SERCA2a thereby reducing intracellular calcium concentration during calcium overload [30]. Thus, TNF- $\alpha$ signaling helps in pumping calcium ions in the sarcoplasmic reticulum and restoring cytosolic calcium back to baseline levels [30]. It is possible that the reduction of TNF- $\alpha$ levels due to GBE observed in the present study may be as a consequence of: (a) reduced expression of the TNF- $\alpha$ gene and a lessened production of TNF- $\alpha$ protein, or, (b) increased production of soluble TNF- $\alpha$ receptors, which could bind and inactivate TNF- $\alpha$ [31]. However, the results of our examination of $\mathrm{T}$ cell phenotypes and function and ex-vivo cytokine production showed no differences in the immune response to GBE.

\section{Limitations and Future Opportunities}

This study has certain limitations that should be taken into account while interpreting the observed findings. Despite the study being sufficiently powered for analysis, the sample size may have been too small as the observed effect size was small. A preliminary pharmacokinetic study would have assisted in appropriate dose selection and treatment regimen. In addition, more than once daily administration might have resulted in a sufficient plasma steady state concentration that could have produced a different outcome. Furthermore, pretreatment with GBE may have resulted in an adequate cardiac tissue distribution of polyphenols necessary for potentiating the endogenous antioxidant system during an ischemic insult. The results of our study open up opportunities for further investigation including a comparative study of a polyphenol rich extract from ginseng berries vs. polyphenol rich extract from roots, and other parts of the plant.

\section{Materials and Methods}

\subsection{Ginseng Berry Pulp Extract}

Ginseng berries from three-year-old Panax quinquefolius L. (North American ginseng) were provided by C \& R Atkinson Farms Ltd, St. Williams, ON, Canada. The berries were stored at $-20{ }^{\circ} \mathrm{C}$ until they could be freeze-dried in smaller batches. After freeze-drying berries, the seeds were removed and the pulp was ground to a fine powder. Pulp extracts were prepared in batches at a ratio 
of $10 \mathrm{~g}$ ground pulp per $200 \mathrm{~mL}$ of $80 \%$ methanol. The slurry was mixed at room temperature using a rotary shaker, at $200 \mathrm{rpm}$ for $1.5 \mathrm{~h}$. The mixture was centrifuged at room temperature for $15 \mathrm{~min}$ at $10,000 \times g$, saving the pellet for further extraction. The supernatant was collected and stored at $-20{ }^{\circ} \mathrm{C}$ and the remaining pulp went through two additional rounds of extraction with $80 \%$ methanol. All supernatants were pooled together and stored at $-20^{\circ} \mathrm{C}$. The solvent was removed by rotary evaporation and the remaining aqueous extract was freeze-dried. Multiple extracts were performed to produce sufficient ginseng berry pulp extract for the study. Dried extracts were further ground to a fine powder and combined to form a homogeneous product for the study. The $\mathrm{pH}$ of the dried extract was 4.5 and the final product was stored at $-80^{\circ} \mathrm{C}$

\subsection{Quantification of Phenolic Content and Antioxidant Activity}

To ensure complete mixing of dried samples three biological replicates were prepared. A quantity of $0.125 \mathrm{~g}$ dried powder of each of these replicates $(\mathrm{a}, \mathrm{b}$, and $\mathrm{c}$ ) was dissolved in $3 \mathrm{~mL}$ of autoclaved 18 ' $\Omega$ Milli-Q water. Tubes were vortexed, sonicated for $10 \mathrm{~min}$ and then centrifuged at $10,000 \times g$. The supernatant was used for subsequent measurement. The assay was performed in a 96 well plate by combining reagent and sample in sodium carbonate buffer. By using a protocol modified from Ainsworth and Gillespie [32], the total phenolic content was determined by spectrometry using SpectraMax M5 (Molecular Devices, San Jose, CA, USA) microplate reader at $765 \mathrm{~nm}$ wavelength. A method modified from Gillespie et al [33] was used to determine the oxygen radical absorbance capacity (ORAC) using 2,2-azobis(2-amidinopropane) dihydrochloride (Wako Chemicals, Richmond, VA, USA) as a peroxyl generator. The decline in fluorescence of fluorescein was measured kinetically for 60 min using a SpectraMax M5 microplate reader and the area under the curve was calculated for each sample.

\subsection{Proximate Analysis}

The core proximate and dietary chemical composition of the sample was determined using standard AOAC methods for ash (AOAC 923.03), fat (AOAC Am5-04), crude fiber (AOAC Ba 6a-05), crude protein modification (AOAC 990.03) and moisture (AOAC 930.15). All tests were conducted by the Central Testing Laboratory (Winnipeg, MB, Canada).

\subsection{Animal Study}

This study protocol was approved by the University of Manitoba Office of Research Ethics and Compliance and Animal Care Committee and was done in accordance with the guidelines by the Canadian Council for Animal Care. Male Sprague Dawley rats (150-175 g; Charles River Laboratories, Quebec, Canada) were housed in a temperature and humidity controlled room with a $12 \mathrm{~h} \mathrm{light/dark}$ cycle. Rats were anesthetized with 1-5\% isoflurane with oxygen at a flow rate of $2 \mathrm{~L} \mathrm{~min}^{-1}$ and kept in a surgical plane of anesthesia with $2 \%$ isoflurane during surgery and subjected to permanent ligation of the left anterior descending artery (LAD) to induce MI or to sham surgery. A left thoracotomy was done, and the heart was gently exposed from the pericardial sac through the incision. The left anterior descending coronary artery (LAD) was located and occluded with 6-0 polypropylene silk suture at about $2 \mathrm{~mm}$ from aortic root. The suture was tied and the ligation was estimated to be successful when the anterior wall of the left ventricle turned pale. The heart was repositioned, then chest compressed to remove any air from the cavity and the incision was closed using a purse string suture. Sham-operated animals that served as normal control were subjected to similar surgical procedures except that the LAD was not ligated. Buprenorphine $0.05 \mathrm{mg} \mathrm{kg}^{-1}$ was administered pre- and post-surgery (2 times a day for 2 days) subcutaneously as an analgesic agent to all rats. All surviving sham and MI rats were assigned to the following 4 treatment groups: (1) Sham MI—distilled water as vehicle (Sham-W); (2) MI—distilled water as vehicle; (MI-W); (3) Sham MI—ginseng berry extract $150 \mathrm{mg} / \mathrm{kg} /$ body weight/day (Sham-G); (4) MI—ginseng berry extract $150 \mathrm{mg} / \mathrm{kg} /$ body weight/day (MI-G). The 4 groups consisted of sham $(n=8)$ and MI $(n=12-14)$ rats and received the 
treatments by oral gavage for 8 weeks. The sample size was calculated using $\mathrm{G}^{*}$ Power statistical software with the power of study kept at $80 \%$. Animals were regularly monitored for well-being.

\subsubsection{Transthoracic Echocardiography}

All experimental rats were weighed and anesthetized with 3\% isoflurane in a chamber, and then kept under $1.5-2 \%$ isoflurane throughout the procedure. An echocardiogram was obtained at 4 and 8 weeks post-surgery by 2D guided M-mode and Doppler modalities with a $13 \mathrm{MHz}$ probe (Vivid E9; GE Medical Systems, Milwaukee, WI, USA) as described by us earlier [34]. 2D M-mode parasternal short-axis view images were obtained to determine systolic functional parameters such as the percentage of left ventricle (LV) ejection fraction (EF), fractional shortening (FS), and end-systolic volume (ESV) and end-diastolic volume (EDV). Doppler measurements included isovolumic relaxation time (IVRT), mitral valve (MV) E wave, A wave, and E wave deceleration time. The cardiac structural parameters such as interventricular septal thickness (IVS), LV posterior wall thickness (LVPW), and LV internal diameter (LVID) at diastole and systole were determined from parasternal short-axis view images. All echocardiographic images were analyzed to calculate the listed parameters using EchoPAC software (GE Medical Systems, Milwaukee, WI, USA). All measurements were performed and averaged from three cardiac cycles to account for interbeat variability.

\subsubsection{Biological Sample Collection and Analysis}

All animals were anesthetized with ketamine/xylazine (9.0 mg per $100 \mathrm{~g}$ and $0.9 \mathrm{mg}$ per $100 \mathrm{~g} \mathrm{IM}$ ). The depth of anesthesia was assessed by pedal withdrawal reflex. The blood sample was collected from the inferior vena cava by opening the thoracic cavity and the heart was immediately excised. The whole heart was rinsed in PBS, LVs, septum, and fibrotic scar tissues were separated, weighed and flash frozen in liquid nitrogen.

Percentage of infarct (scarred/fibrotic) LV tissue was calculated by dividing the weight of scarred LV tissue by whole weight of LV tissue as described previously [35]. Evidence of overt heart failure was assessed by determining the presence of ascites and by calculating the lung and liver wet-to-dry weight ratio in all rats.

To determine MI-associated oxidative stress and inflammation, the levels of the lipid peroxidation product, malondialdehyde (MDA), and tumor necrosis factor- $\alpha$ (TNF $\alpha$ ) as a proinflammatory marker were assessed in the heart tissue using commercial kits.

\subsection{T-cell Phenotypes and Function}

To assess the effect of MI and GBE treatment on T-cell phenotypes and activation state and T-cell function, the following experiments were conducted.

\subsubsection{Isolation of T-cells}

Single cell suspensions were obtained by pressing spleens through a $100 \mu \mathrm{m}$ cell strainer using the barrel of a sterile syringe into a sterile Petri dish containing Hank's buffered saline supplemented with $10 \mathrm{mM}$-HEPES, $4 \%$ fetal bovine serum, and $1 \%$ antibiotic/antimycotic at $\mathrm{pH}$ 7.4. Erythrocytes were lysed with ammonium chloride buffer. Cells were subsequently washed and re-suspended in label buffer (PBS containing $23 \mathrm{mM}$-sodium azide and $2 \%$ fetal bovine serum). Cell count and viability was completed using a Nexcelom AutoT4Plus. Cell concentration was adjusted to $1 \times 10^{7}$ cells $/ \mathrm{mL}$ in label buffer [36].

\subsubsection{Cytokine Determination}

Splenocytes were resuspended in RPMI-1640 supplemented with 10mM-HEPES, 10 mM-sodium bicarbonate, $1 \mathrm{mM}$-sodium pyruvate, $2 \mathrm{mM}$-gluatmine, $0.1 \mathrm{mM}$-non-essential amino acids, $50 \mu \mathrm{M}$-2-mercatpoethanol, $1 \%$ antibiotic/antimycotic and 5\% fetal bovine serum at $\mathrm{pH} 7.2$ at a 
concentration of $1 \times 10^{6}$ splenocytes $/ \mathrm{mL}$ and incubated at $37^{\circ} \mathrm{C}$ and $5 \% \mathrm{CO}_{2}$ with $2.5 \mu \mathrm{g} / \mathrm{mL}$ Concanavalin A (ConA), or unstimulated for 48 hours. After incubation, samples were centrifuged at $400 \mathrm{~g}$ at $4{ }^{\circ} \mathrm{C}$ for $5 \mathrm{~min}$ to pellet cells. Supernatants were stored at $-80{ }^{\circ} \mathrm{C}$ until analysis. The concentration of IL-2 (lower limit of detection (LLOD) $0.46 \mathrm{pg} / \mathrm{mL}$ ), IFN $\gamma$ (LLOD $6.8 \mathrm{pg} / \mathrm{mL}$ ), IL-10 (LLOD $19.4 \mathrm{pg} / \mathrm{mL}$ ), and TNF $\alpha$ (LLOD $27.7 \mathrm{pg} / \mathrm{mL}$ ) were measured in cell culture supernatants using a cytometric bead array on a FACSCanto II flow cytometer. All samples were analyzed in duplicate with CV $<10 \%$ according to the BD Cytometric Bead Array Mouse/Rat Soluble Protein Master Buffer Kit Instruction Manual [37].

\subsubsection{Phenotyping}

T-cell phenotypes were determined by flow cytometry using isolated splenocytes. Monoclonal antibodies against rat CD3 (FITC label, clone 1F4, isotype mouse $\mathrm{IgM}_{\mathrm{K}}$ ), CD4 (PE-Cy7 label, clone OX-35, isotype mouse $\operatorname{Ig}_{2 \mathrm{a}, \mathrm{k}}$ ), CD8 (PerCP label, clone OX-8, isotype mouse $\operatorname{IgG}_{1, \mathrm{k}}$ ), CD25 (APC label, clone OX39, isotype Mouse $\operatorname{IgG}_{1, \kappa}$ ), foxp3 (PE label, clone FJK-16s, isotype rat $\operatorname{IgG}_{2 a, k}$ ) were obtained from BD BioSciences (Mississauga, ON, Canada). Antibodies were incubated with $1 \times 10^{6}$ cells $/ \mathrm{mL}$ for $30 \mathrm{~min}$ at $4{ }^{\circ} \mathrm{C}$ in the dark. Cells were washed, and then incubated in Foxp3 fixation/permeabilization working solution at $4{ }^{\circ} \mathrm{C}$ for $18 \mathrm{~h}$. Following incubation cells were washed with the permeabilization buffer and incubated with mouse CD16/CD32 antibody at $4{ }^{\circ} \mathrm{C}$ for $5 \mathrm{~min}$ to block non-specific binding. Foxp3 was added to cells and incubated at $4{ }^{\circ} \mathrm{C}$ in the dark for at least $30 \mathrm{~min}$. Finally, treated cells were washed using the permeabilization buffer and re-suspended in PBS. Data were acquired on a FACSCanto II flow cytometer using the $488 \mathrm{~nm}$ and $633 \mathrm{~nm}$ lasers. Figure 6 shows representative flow cytometry plots. Forward versus side-scatter plots were used to gate on intact lymphoid cells and non-viable cells. The data were collected in list-mode format with the analyses based on 100,000 cells satisfying the light scatter gate for lymphocytes using Cell Diva software (v8.0.1). Unstained cells were used to assess auto-fluorescence, isotype controls to assess background staining, and single color samples were employed to adjust color compensation [36].

\subsection{Statistical Analysis}

All values are represented as means \pm SEM. Two-way analysis of variance (ANOVA) test was used to determine the effect of surgery (factor 1) and treatment (factor 2 ) and their interaction (SAS, version 9.4, SAS Institute, Cary NC, USA). Unpaired t-test was utilized for comparison between 2 groups Significant differences among means were determined using LSmeans. Differences were considered significant at $p \leq 0.05$.

\section{Conclusions}

The results of this study suggest that although GBE exerted potent antioxidant activity, it was unable to recover cardiac function in post-MI rats.

Author Contributions: The authors were responsible for the following aspects of this paper: conceptualization, T.N.; methodology, T.N., Y.S., and H.B.; formal analysis, M.P.; investigation, P.R., J.S., S.P., J.C.P., and L.Y.; resources, D.B., T.N.; data curation, L.Y.; writing-original draft preparation, M.P., P.R, H.B; writing-review and editing, M.P, P.R., G.N.P., Y.S., H.B., P.S.T., J.S., S.P., J.C.P., and T.N.; supervision, T.N.; project administration, L.Y., and T.N.; funding acquisition, D.B, Y.S., T.N., and H.B.

Funding: This research was funded by Agriculture and Agri-Food Canada.

Acknowledgments: We would like to thank the R.O. Burrell Laboratory for assistance with animal studies.

Conflicts of Interest: The authors declare no conflict of interest. 


\section{References}

1. Vuksan, V.; Xu, Z.Z.; Jovanovski, E.; Jenkins, A.L.; Beljan-Zdravkovic, U.; Sievenpiper, J.L.; Mark Stavro, P.; Zurbau, A.; Duvnjak, L.; Li, M.Z.C. Efficacy and safety of American ginseng (Panax quinquefolius L.) extract on glycemic control and cardiovascular risk factors in individuals with type 2 diabetes: A double-blind, randomized, cross-over clinical trial. Eur. J. Nutr. 2018. [CrossRef] [PubMed]

2. Attele, A.S.; Wu, J.A.; Yuan, C.-S. Ginseng pharmacology: Multiple constituents and multiple actions. Biochem. Pharmacol. 1999, 58, 1685-1693. [CrossRef]

3. Chung, I.M.; Lim, J.J.; Ahn, M.S.; Jeong, H.N.; An, T.J.; Kim, S.H. Comparative phenolic compound profiles and antioxidative activity of the fruit, leaves, and roots of Korean ginseng (Panax ginseng Meyer) according to cultivation years. J. Ginseng Res. 2016, 40, 68-75. [CrossRef] [PubMed]

4. Mehendale, S.R.; Wang, C.Z.; Shao, Z.H.; Li, C.Q.; Xie, J.T.; Aung, H.H.; Yuan, C.S. Chronic pretreatment with American ginseng berry and its polyphenolic constituents attenuate oxidant stress in cardiomyocytes. Eur. J. Pharmacol. 2006, 553, 209-214. [CrossRef] [PubMed]

5. Choi, S.Y.; Cho, C.W.; Lee, Y.; Kim, S.S.; Lee, S.H.; Kim, K.T. Comparison of Ginsenoside and Phenolic Ingredient Contents in Hydroponically-cultivated Ginseng Leaves, Fruits, and Roots. J. Ginseng Res. 2012, 36, 425-429. [CrossRef] [PubMed]

6. Hyun, T.K.; Jang, K.I. Are berries useless by-products of ginseng? Recent research on the potential health benefits of ginseng berry. Excli J. 2017, 16, 780-784. [PubMed]

7. Dey, L.; Xie, J.T.; Wang, A.; Wu, J.; Maleckar, S.A.; Yuan, C.S. Anti-hyperglycemic effects of ginseng: Comparison between root and berry. Phytomedicine 2003, 10, 600-605. [CrossRef] [PubMed]

8. Kim, M.H.; Lee, J.; Jung, S.; Kim, J.W.; Shin, J.H.; Lee, H.J. The involvement of ginseng berry extract in blood flow via regulation of blood coagulation in rats fed a high-fat diet. J. Ginseng Res. 2017, 41, 120-126. [CrossRef] [PubMed]

9. Seo, E.; Kim, S.; Lee, S.J.; Oh, B.C.; Jun, H.S. Ginseng berry extract supplementation improves age-related decline of insulin signaling in mice. Nutrients 2015, 7, 3038-3053. [CrossRef] [PubMed]

10. Choi, H.S.; Kim, S.; Kim, M.J.; Kim, M.S.; Kim, J.; Park, C.W.; Seo, D.; Shin, S.S.; Oh, S.W. Efficacy and safety of Panax ginseng berry extract on glycemic control: A 12-wk randomized, double-blind, and placebo-controlled clinical trial. J. Ginseng Res. 2018, 42, 90-97. [CrossRef] [PubMed]

11. Li, J.; Ichikawa, T.; Jin, Y.; Hofseth, L.J.; Nagarkatti, P.; Nagarkatti, M.; Windust, A.; Cui, T. An essential role of Nrf2 in American ginseng-mediated anti-oxidative actions in cardiomyocytes. J. Ethnopharmacol. 2010, 130, 222-230. [CrossRef] [PubMed]

12. Moey, M.; Gan, X.T.; Huang, C.X.; Rajapurohitam, V.; Martinez-Abundis, E.; Lui, E.M.; Karmazyn, M. Ginseng reverses established cardiomyocyte hypertrophy and postmyocardial infarction-induced hypertrophy and heart failure. Circ. Heart Fail. 2012, 5, 504-514. [CrossRef] [PubMed]

13. Frangogiannis, N.G. Regulation of the inflammatory response in cardiac repair. Circ. Res. 2012, 110, 159-173. [CrossRef] [PubMed]

14. Magrone, T.; Jirillo, E. Polyphenols from red wine are potent modulators of innate and adaptive immune responsiveness. Proc. Nutr. Soc. 2010, 69, 279-285. [CrossRef] [PubMed]

15. Kim, C.-K.; Cho, D.H.; Lee, K.-S.; Lee, D.-K.; Park, C.-W.; Kim, W.G.; Lee, S.J.; Ha, K.-S.; Goo Taeg, O.; Kwon, Y.-G.; Kim, Y.-M. Ginseng Berry Extract Prevents Atherogenesis via Anti-Inflammatory Action by Upregulating Phase II Gene Expression. Evid. -Based Complement. Altern. Med. 2012, 2012. [CrossRef] [PubMed]

16. Attele, A.S.; Zhou, Y.P.; Xie, J.T.; Wu, J.A.; Zhang, L.; Dey, L.; Pugh, W.; Rue, P.A.; Polonsky, K.S.; Yuan, C.S. Antidiabetic effects of Panax ginseng berry extract and the identification of an effective component. Diabetes 2002, 51, 1851-1858. [CrossRef] [PubMed]

17. Li, Z.; Kim, H.J.; Park, M.S.; Ji, G.E. Effects of fermented ginseng root and ginseng berry on obesity and lipid metabolism in mice fed a high-fat diet. J. Ginseng Res. 2018, 42, 312-319. [CrossRef] [PubMed]

18. Shao, Z.-H.; Xie, J.-T.; Vanden Hoek, T.L.; Mehendale, S.; Aung, H.; Li, C.-Q.; Qin, Y.; Schumacker, P.T.; Becker, L.B.; Yuan, C.-S. Antioxidant effects of American ginseng berry extract in cardiomyocytes exposed to acute oxidant stress. Biochim. Biophys. Acta Gen. Subj. 2004, 1670, 165-171. [CrossRef] [PubMed]

19. Eaton, L.W.; Weiss, J.L.; Bulkley, B.H.; Garrison, J.B.; Weisfeldt, M.L. Regional Cardiac Dilatation after Acute Myocardial Infarction. N. Engl. J. Med. 1979, 300, 57-62. [CrossRef] [PubMed] 
20. Erlebacher, J.A.; Weiss, J.L.; Eaton, L.W.; Kallman, C.; Weisfeldt, M.L.; Bulkley, B.H. Late effects of acute infarct dilation on heart size: A two dimensional echocardiographic study. Am. J. Card. 1982, 49, 1120-1126. [CrossRef]

21. Gaudron, P.; Eilles, C.; Kugler, I.; Ertl, G. Progressive left ventricular dysfunction and remodeling after myocardial infarction. Potential mechanisms and early predictors. Circulation 1993, 87, 755-763. [CrossRef] [PubMed]

22. Rigo, P.; Murray, M.; Strauss, H.W.; Taylor, D.; Kelly, D.; Weisfeldt, M.; Pitt, B. Left Ventricular Function in Acute Myocardial Infarction Evaluated by Gated Scintiphotography. Circulation 1974, 50, 678-684. [CrossRef] [PubMed]

23. Moller, J.E.; Hillis, G.S.; Oh, J.K.; Reeder, G.S.; Gersh, B.J.; Pellikka, P.A. Wall motion score index and ejection fraction for risk stratification after acute myocardial infarction. Am. Heart J. 2006, 151, 419-425. [CrossRef] [PubMed]

24. Multicenter Postinfarction Research Group. Risk Stratification and Survival after Myocardial Infarction. N. Engl. J. Med. 1983, 309, 331-336. [CrossRef] [PubMed]

25. White, H.D.; Norris, R.M.; Brown, M.A.; Brandt, P.W.; Whitlock, R.M.; Wild, C.J. Left ventricular end-systolic volume as the major determinant of survival after recovery from myocardial infarction. Circulation 1987, 76, 44-51. [CrossRef] [PubMed]

26. Janahmadi, Z.; Nekooeian, A.A.; Moaref, A.R.; Emamghoreishi, M. Oleuropein Offers Cardioprotection in Rats with Acute Myocardial Infarction. Cardiovasc. Toxicol. 2015, 15, 61-68. [CrossRef] [PubMed]

27. Zhao, H.; Zhang, J.; Hong, G. Minocycline improves cardiac function after myocardial infarction in rats by inhibiting activation of PARP-1. Biomed. Pharmacother. 2018, 97, 1119-1124. [CrossRef] [PubMed]

28. Shanmugam, G.; Narasimhan, M.; Sakthivel, R.; Kumar, R.R.; Davidson, C.; Palaniappan, S.; Claycomb, W.W.; Hoidal, J.R.; Darley-Usmar, V.M.; Rajasekaran, N.S. A biphasic effect of TNF-alpha in regulation of the Keap1/Nrf2 pathway in cardiomyocytes. Redox Biol. 2016, 9, 77-89. [CrossRef] [PubMed]

29. Kurrelmeyer, K.M.; Michael, L.H.; Baumgarten, G.; Taffet, G.E.; Peschon, J.J.; Sivasubramanian, N.; Entman, M.L.; Mann, D.L. Endogenous tumor necrosis factor protects the adult cardiac myocyte against ischemic-induced apoptosis in a murine model of acute myocardial infarction. Proc. Natl. Acad. Sci. USA 2000, 97, 5456-5461. [CrossRef] [PubMed]

30. El-Ani, D.; Philipchik, I.; Stav, H.; Levi, M.; Zerbib, J.; Shainberg, A. Tumor necrosis factor alpha protects heart cultures against hypoxic damage via activation of PKA and phospholamban to prevent calcium overload. Can. J. Physiol. Pharmacol. 2014, 92, 917-925. [CrossRef] [PubMed]

31. Utheim, T.P. Chapter 7: Why Test BCG in Sjögren's Syndrome? In The Value of BCG and TNF in Autoimmunity; Faustman, D.L., Ed.; Academic Press: Amsterdam, The Netherlands, 2014; pp. 105-125.

32. Ainsworth, E.A.; Gillespie, K.M. Estimation of total phenolic content and other oxidation substrates in plant tissues using Folin-Ciocalteu reagent. Nat. Protoc. 2007, 2, 875-877. [CrossRef] [PubMed]

33. Gillespie, K.M.; Chae, J.M.; Ainsworth, E.A. Rapid measurement of total antioxidant capacity in plants. Nat. Protoc. 2007, 2, 867-870. [CrossRef] [PubMed]

34. Raj, P.; McCallum, J.L.; Kirby, C.; Grewal, G.; Yu, L.; Wigle, J.T.; Netticadan, T. Effects of cyanidin 3-0-glucoside on cardiac structure and function in an animal model of myocardial infarction. Food Funct. 2017, 8, 4089-4099. [CrossRef] [PubMed]

35. Ju, H.; Zhao, S.; Jassal, D.S.; Dixon, I.M. Effect of AT1 receptor blockade on cardiac collagen remodeling after myocardial infarction. Cardiovasc. Res. 1997, 35, 223-232. [CrossRef]

36. Blewett, H.J.; Mohankumar, S.K.; Rech, L.; Rector, E.S.; Taylor, C.G. The reduced proportion of New splenic T-cells in the zinc-deficient growing rat is not due to increased susceptibility to apoptosis. Immunobiology 2014, 219, 602-610. [CrossRef] [PubMed]

37. Blewett, H.J.; Gerdung, C.A.; Ruth, M.R.; Proctor, S.D.; Field, C.J. Vaccenic acid favourably alters immune function in obese JCR:LA-cp rats. Br. J. Nutr. 2009, 102, 526-536. [CrossRef] [PubMed]

(C) 2019 by the authors. Licensee MDPI, Basel, Switzerland. This article is an open access article distributed under the terms and conditions of the Creative Commons Attribution (CC BY) license (http:/ / creativecommons.org/licenses/by/4.0/). 